\title{
CMS studies in heavy flavor production
}

\author{
Mario Galanti ${ }^{* \dagger}$ \\ Department of Physics and Astronomy, University of Rochester \\ 206 Bausch and Lomb Hall, P.O. Box 270171 \\ Rochester, NY 14627-0171 (USA) \\ E-mail: mario.galanti@cern.ch
}

In these proceedings I shortly describe some of the most recent results published by the CMS Collaboration in the field of heavy flavor production. In particular, I discuss measurements of open heavy flavor and quarkonium production cross sections, and of heavy baryon polarization at $\sqrt{s}=7,8$, and $13 \mathrm{TeV}$.

18th International Conference on B-Physics at Frontier Machines - Beauty2019 -

29 September $/ 4$ October, 2019

Ljubljana, Slovenia

\footnotetext{
* Speaker.

${ }^{\dagger}$ On behalf of the CMS Collaboration.
} 


\section{Introduction}

In these proceedings I shortly describe some of the most recent results published by the CMS Collaboration in the field of heavy flavor production. A detailed description of the CMS detector can be found in Ref. [1]. The analyses shown in the following sections use proton-proton collision data collected by the CMS experiment at the CERN LHC from 2011 to 2018 at center-of-mass energies of 7, 8 , and $13 \mathrm{TeV}$, and include both cross-section and polarization measurements.

The proceedings are organized in two parts, dedicated respectively to open heavy flavor and quarkonium results.

\section{Measurements of open heavy flavor production}

\section{1 $\mathrm{B}^{+}$cross section at $13 \mathrm{TeV}$}

CMS measured the $\mathrm{B}^{+}$cross section in pp collisions at $13 \mathrm{TeV}$ using $48.1 \mathrm{pb}^{-1}$ of data collected at low instantaneous luminosity [2]. The measurement is based on the inclusive channel $\mathrm{pp} \rightarrow \mathrm{B}^{+} X \rightarrow \mathrm{J} / \psi \mathrm{K}^{+} X$, with $\mathrm{J} / \psi \rightarrow \mu^{+} \mu^{-}$, and is conducted in the fiducial phase space defined by: $\left|\mathrm{y}\left(\mathrm{B}^{+}\right)\right|<1.45$ for $10 \leq p_{\mathrm{T}}\left(\mathrm{B}^{+}\right)<17 \mathrm{GeV}$ and $\left|\mathrm{y}\left(\mathrm{B}^{+}\right)\right|<2.1$ for $17 \leq p_{\mathrm{T}}\left(\mathrm{B}^{+}\right)<100 \mathrm{GeV}$.

The main background contributions are from combinatorial dimuons, $\mathrm{B}^{+} \rightarrow \mathrm{J} / \psi \pi^{+}$, and $\mathrm{B}^{+} \rightarrow \mathrm{J} / \psi+$ hadrons decays. The signal component is extracted through a maximum-likelihood fit to the $\mathrm{J} / \psi \mathrm{K}^{+}$invariant mass distribution.

The main sources of systematic uncertainties are the muon trigger, identification, and reconstruction, the likelihood fit model, and the kinematic distributions of $\mathrm{B}^{+}$events in simulation. The results are not statistically-limited.

The differential $\mathrm{B}^{+}$production cross-sections are extracted in bins of $p_{\mathrm{T}}\left(\mathrm{B}^{+}\right)$and $\left|\mathrm{y}\left(\mathrm{B}^{+}\right)\right|$and can be seen in Fig. 1.
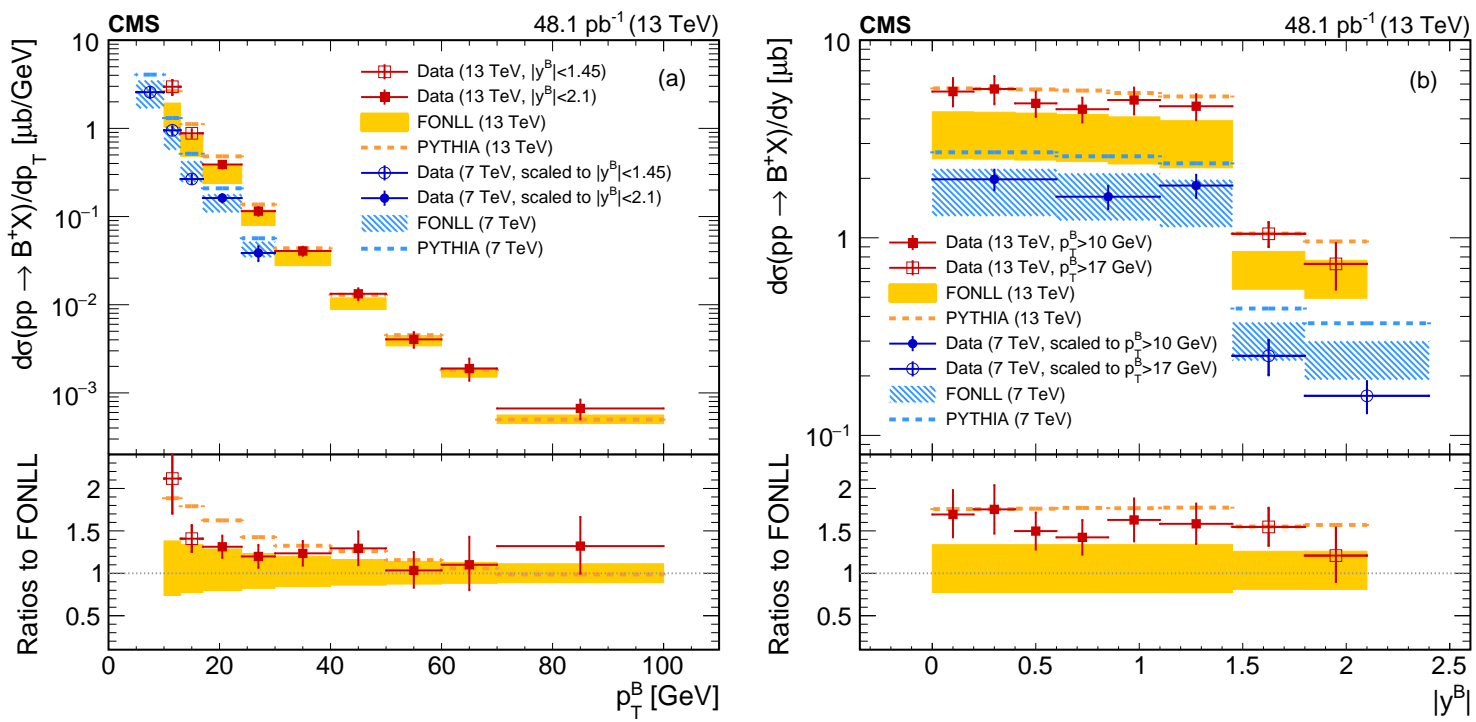

Figure 1: The $\mathrm{B}^{+}$differential production cross sections at $\sqrt{s}=13 \mathrm{TeV}$ measured by $\mathrm{CMS}$ as a function of $p_{\mathrm{T}}\left(\mathrm{B}^{+}\right)$(left) and $\left|\mathrm{y}\left(\mathrm{B}^{+}\right)\right|$(right) [2], compared with the theoretical predictions and with $7 \mathrm{TeV}$ results [3]. 
The cross sections measured by CMS at $\sqrt{s}=13 \mathrm{TeV}$ are compared with Pythia [4] and FONLL [5,6] predictions and with CMS results at $7 \mathrm{TeV}$ [3], rescaled to match the same phase space of the new measurement. CMS data at $\sqrt{s}=13 \mathrm{TeV}$ (red points) are compatible with FONLL (yellow bands) at high $p_{\mathrm{T}}\left(\mathrm{B}^{+}\right)$while they tend to favor higher cross sections at low $p_{\mathrm{T}}\left(\mathrm{B}^{+}\right)$. Results at $7 \mathrm{TeV}$ are in good agreement with FONLL (blue bands) at low $p_{\mathrm{T}}\left(\mathrm{B}^{+}\right)$.

The total $\mathrm{B}^{+}$cross section at $\sqrt{s}=13 \mathrm{TeV}$ in the fiducial phase space is extracted from the sum over all bins and is found to be $\sigma\left(p p \rightarrow \mathrm{B}^{+}+X\right)=15.3 \pm 0.4$ (stat) \pm 2.1 (syst) \pm 0.4 (lumi) pb.

\section{$2.2 \Lambda_{\mathrm{b}}^{0}$ polarization at 7 and $8 \mathrm{TeV}$}

The polarization parameters of $\Lambda_{\mathrm{b}}$ baryons were measured by CMS on $5.3 \mathrm{fb}^{-1}$ of $7 \mathrm{TeV}$ and $19.8 \mathrm{fb}^{-1}$ of $8 \mathrm{TeV}$ pp data [7], through the decay $\Lambda_{\mathrm{b}} \rightarrow \mathrm{J} / \psi\left(\rightarrow \mu^{+} \mu^{-}\right) \Lambda\left(\rightarrow \mathrm{p} \pi^{-}\right)$. This decay can be described as a function of the helicity amplitudes $T_{\lambda_{1}} \lambda_{2}$, where $\lambda_{1}$ and $\lambda_{2}$ refer to the helicities of the $\Lambda$ and $\mathrm{J} / \psi$ particles, respectively. Four physical parameters of interest are measured by CMS, which depend on $T_{\lambda_{1} \lambda_{2}}$ : the $\Lambda_{\mathrm{b}}$ polarization $P$, the asymmetry in $\Lambda_{\mathrm{b}} \rightarrow \mathrm{J} / \psi \Lambda$ decay $\alpha_{1}$, the longitudinal $\Lambda$ polarization $\alpha_{2}$, and the parameter $\gamma_{0}$, which is related to the $\mathrm{J} / \psi$ longitudinal and transverse polarizations.

The four parameters $\left(P, \alpha_{1}, \alpha_{2}, \gamma_{0}\right)$ are measured through an unbinned maximum likelihood fit to the invariant mass $m(\mathrm{~J} / \psi \Lambda)$ and the three angular parameters $\Theta_{3}=\left(\cos \left(\theta_{\Lambda}\right), \cos \left(\theta_{\mathrm{p}}\right), \cos \left(\theta_{\mu}\right)\right)$. The three angles appearing in $\Theta_{3}$ are defined as shown in Fig. 2.

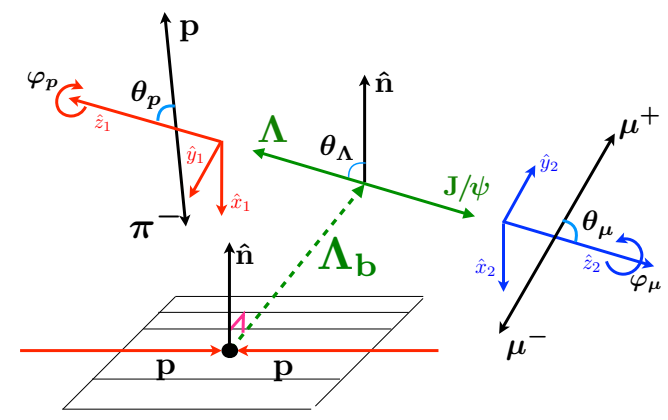

Figure 2: A graphical depiction of the $\Lambda_{\mathrm{b}} \rightarrow \mathrm{J} / \psi\left(\rightarrow \mu^{+} \mu^{-}\right) \Lambda\left(\rightarrow \mathrm{p} \pi^{-}\right)$decay, showing the definition of the three angles $\theta_{\Lambda}, \theta_{\mathrm{p}}$, and $\theta_{\mu}$ [7].

The fit is performed separately for $\Lambda_{\mathrm{b}}$ and $\bar{\Lambda}_{\mathrm{b}}$ candidates. As an illustration, Fig. 3 shows the distributions of the four variables $m(\mathrm{~J} / \psi \Lambda)$ and $\Theta_{3}$ for the $\Lambda_{\mathrm{b}}$ candidates with the fit results superimposed.

The analysis precision is limited by the statistical uncertainty. The main systematic uncertainty is related to the reconstruction bias, evaluated in the simulation by comparing the generated and measured values of the polarization parameters.

The four physical parameters are measured to be:

$$
\begin{aligned}
P & =0.00 \pm 0.06(\text { stat }) \pm 0.06(\text { syst }) \\
\alpha_{1} & =0.14 \pm 0.14(\text { stat }) \pm 0.10(\text { syst }) \\
\alpha_{2} & =-1.11 \pm 0.04(\text { stat }) \pm 0.05(\text { syst }) \\
\gamma_{0} & =-0.27 \pm 0.08(\text { stat }) \pm 0.11 \text { (syst) } .
\end{aligned}
$$



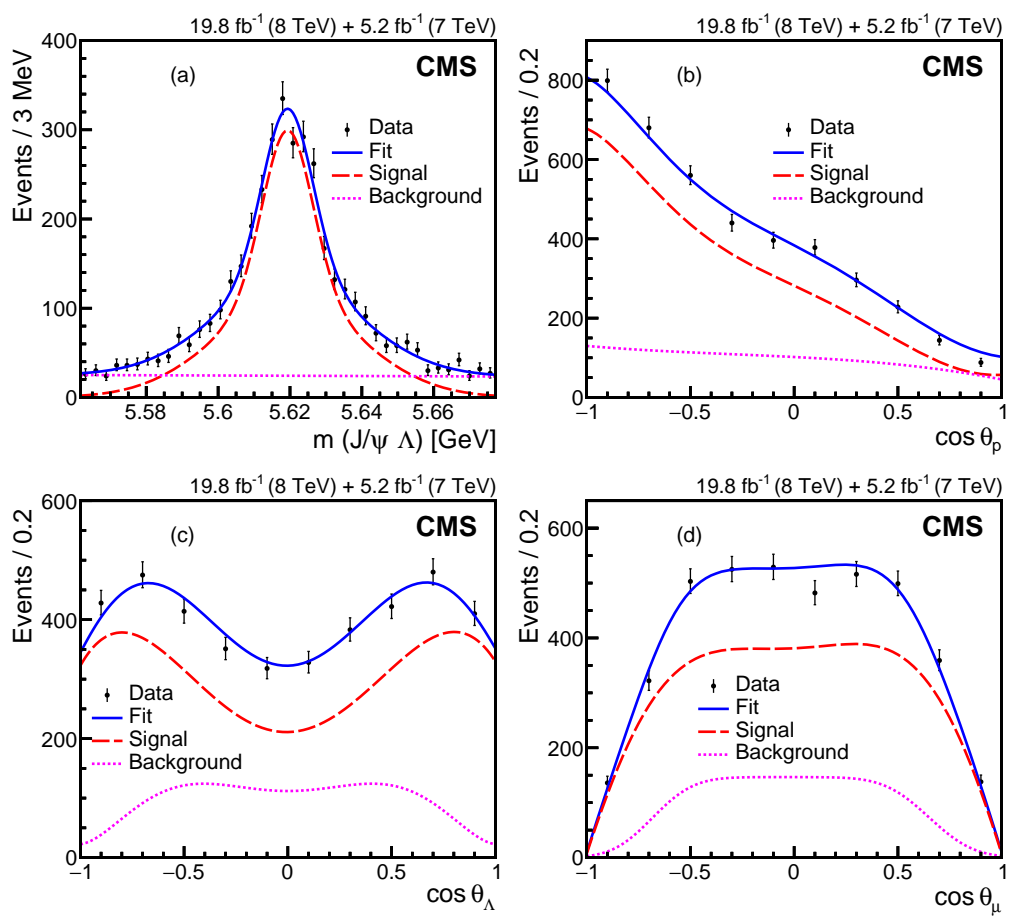

Figure 3: The distributions of the four variables $m(\mathrm{~J} / \psi \Lambda)$ and $\Theta_{3}=\left(\cos \left(\theta_{\Lambda}\right), \cos \left(\theta_{\mathrm{p}}\right), \cos \left(\theta_{\mu}\right)\right)$ for the case of the $\Lambda_{\mathrm{b}}$ candidates. The results of the fit used to extract the $\Lambda_{\mathrm{b}}$ polarization parameters are superimposed [7].

The measured values are inconsistent at more than 5 standard deviations with the heavy-quark effective theory prediction [8,9], but are consistent at less than one standard deviation with other predictions from quark model techniques [10-14] and from perturbative quantum chromodymamics calculations [15]. These values are also consistent with previous measurements by LHCb [16] and ATLAS [17].

\section{Measurements of quarkonium production}

\section{1 $\mathrm{J} / \psi$ production from jet fragmentation}

The production of $\mathrm{J} / \psi$ mesons within jets can be used to probe the long-distance matrix elements (LDMEs) contributions to jet fragmentation. CMS studied it on $19.1 \mathrm{fb}^{-1}$ of pp collisions at $\sqrt{s}=8 \mathrm{TeV}$, considering the possible contributions from four different LDMEs: ${ }^{1} S_{0}^{(8)},{ }^{3} S_{1}^{(8)},{ }^{3} S_{1}^{(1)}$, and ${ }^{3} P_{j}^{(8)}[18,19]$.

The measurement is conducted in different bins of the $\mathrm{J} / \psi$ energy fraction $z$, defined as:

$$
z \equiv \mathrm{E}(\mathrm{J} / \psi) / \mathrm{E}(\mathrm{jet})
$$

The sensitive variable used to disentangle the different LDMEs contributions is $\Xi\left(\mathrm{E}_{1}, z_{1}\right)$ :

$$
\left.\Xi\left(\mathrm{E}_{1}, z_{1}\right) \equiv \frac{N\left(\mathrm{E}_{1}, z_{1}\right)}{\int_{0.3}^{0.8} N\left(\mathrm{E}_{1}, z\right) \mathrm{d} z} \equiv \frac{\mathrm{d} \widetilde{\sigma}}{\mathrm{dEd} z}\right|_{\mathrm{E}_{1}, z_{1}} .
$$


The second term of Eq. (3.1) describes how $\Xi\left(\mathrm{E}_{1}, z_{1}\right)$ can be measured experimentally, with $N\left(\mathrm{E}_{1}, z_{1}\right)$ being the number of events having jet energy $\mathrm{E}_{1}$ and $z$ within an interval $\Delta_{z}= \pm 0.025$ around $z_{1}$. The integration interval has been chosen in such a way as to be within the acceptance of the CMS detector.

To measure $\Xi\left(\mathrm{E}_{1}, z_{1}\right)$, the $\mathrm{J} / \psi$ mesons reconstructed by CMS are matched to jets in $\Delta R$, considering both single- and double-jets events. Data distributions are corrected for efficiencies and unfolded with the D'Agostini method from the RooUnfold package [20]. Three $z$ bins are considered: $0.4<z<0.45,0.5<z<0.55$, and $0.6<z<0.65$. The main systematic uncertainties of the measurement are related to the efficiency determination and to the association between $\mathrm{J} / \psi$ mesons and jets.

The third term of Eq. (3.1) depends on theoretical predictions. In general, this term is the sum of the contributions of all the LDMEs to the differential cross section, including interferences. However, if just one LDME dominates at a given $z$ value, then one can obtain a good description of the jet energy dependence in data by just considering its contribution alone in Eq. (3.1). Otherwise, it is unlikely that any single LDME will be a good match for the energy dependence observed in data.

The theoretical predictions in the last term of Eq. (3.1) are obtained within the Fragmenting Jet Function (FJF) approach [21], with two different sets of LDMEs being tested, respectively from Bodwin, Chung, Kim, and Lee (BCKL) [22], and from Butenschoen and Kniehl (BK) [23].

The prediction of each LDME is tested against the CMS results by evaluating the $\chi^{2}$ and $\chi^{2}$ probabilities of the match between the distributions of data points and the theory curves as a function of $\mathrm{E}$ (jet) in each $z$ bin. The CMS results for the first $z$ bin, compared with the theoretical predictions for the 8 LDMEs tested, can be seen in Fig. 4.
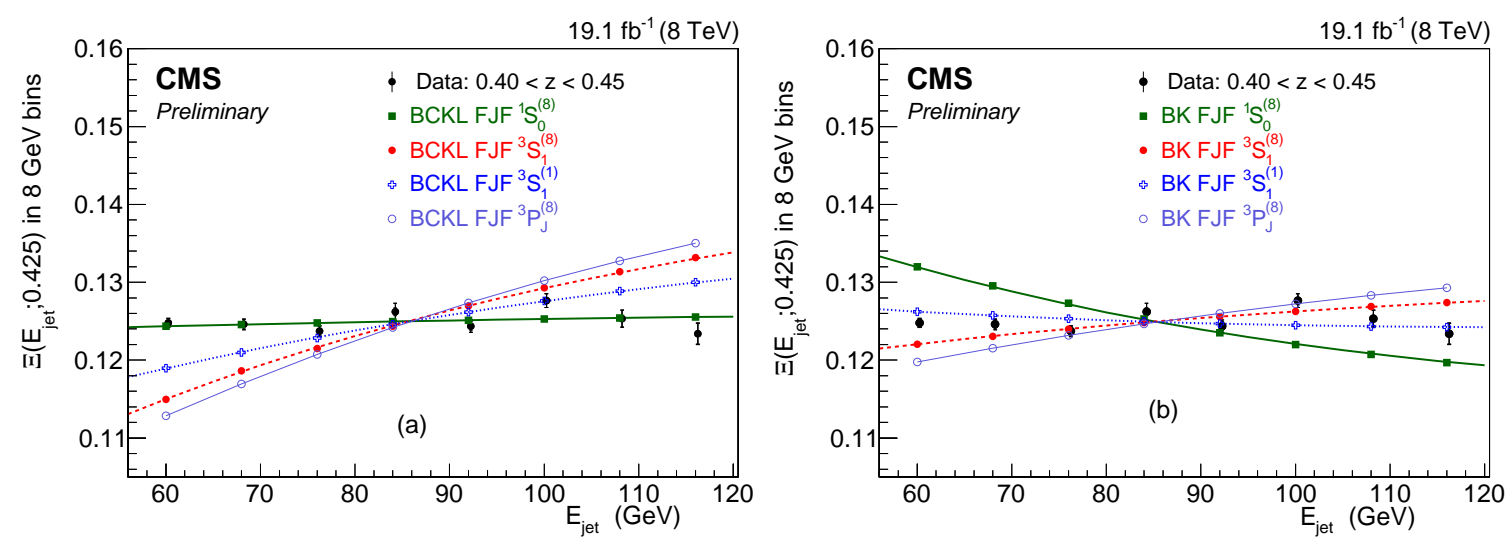

Figure 4: The $\Xi\left(\mathrm{E}_{1}, z_{1}\right)$ distribution for the $0.4<z<0.45$ bin measured by CMS as a function of $\mathrm{E}$ (jet) [18], compared with the theoretical predictions from the BCKL (left) and BK (right) LDME sets.

In general, one finds that the only LDME providing an acceptable match to the CMS data distributions in all $z$ bins is $\mathrm{BCKL}^{1} S_{0}^{(8)}$, with $\chi^{2}$ probabilities in the range between $4.6 \%$ and $18 \%$. The ${ }^{3} S_{1}^{(8)}$ and ${ }^{3} P_{j}^{(8)}$ LDMEs are highly disfavored at all $z$ for both sets. For the second and third $z$ bin, the ${ }^{3} S_{1}^{(1)}$ LDME of the BK set also provides acceptable matches to the data points, creating a possible degeneracy between this term and BCKL ${ }^{1} S_{0}^{(8)}$ for $z>0.5$. However, the ${ }^{3} S_{1}^{(1)}$ LDME 
is disfavored by past polarization measurements (see e.g. Refs. [24, 25]). In fact, a dominance of this term would imply a significant transverse polarization of the $\mathrm{J} / \psi$ meson, which has never been observed in data.

\subsection{Quarkonium production at central rapidity}

The production cross sections of $\mathrm{J} / \psi, \psi(2 \mathrm{~S})$, and $\mathrm{Y}(n \mathrm{~S})$, with $n=(1,2,3)$, at central rapidity $(|y|<1.2)$ were measured by CMS on 2.3-2.7 $\mathrm{fb}^{-1}$ of pp data at $\sqrt{s}=13 \mathrm{TeV}$ [26]. All quarkonium states are reconstructed through their decays to $\mu^{+} \mu^{-}$pairs. The analysis measures both singleand double-differential cross sections times branching fractions as a function of $p_{\mathrm{T}}$ and rapidity for the five quarkonia considered.

The signal yields are obtained through an extended unbinned maximum-likelihood fit to the dimuon invariant mass distribution in the case of the $\mathrm{Y}(n \mathrm{~S})$ states, and to the dimuon invariant mass and the decay length distributions for the $\mathrm{J} / \psi$ and $\psi(2 \mathrm{~S})$ mesons. The raw yields returned by the fit are then corrected for acceptance and efficiency.

Cross section results are shown in Fig. 5, compared with non-relativistic QCD (NRQCD) predictions [27,28] and with past CMS measurements at $\sqrt{s}=7 \mathrm{TeV}[29,30]$. CMS data is in reasonable agreement with NRQCD predictions, within uncertainties. The $13 \mathrm{TeV} / 7 \mathrm{TeV}$ ratios show a slight increase with $p_{\mathrm{T}}$ for all quarkonium species.
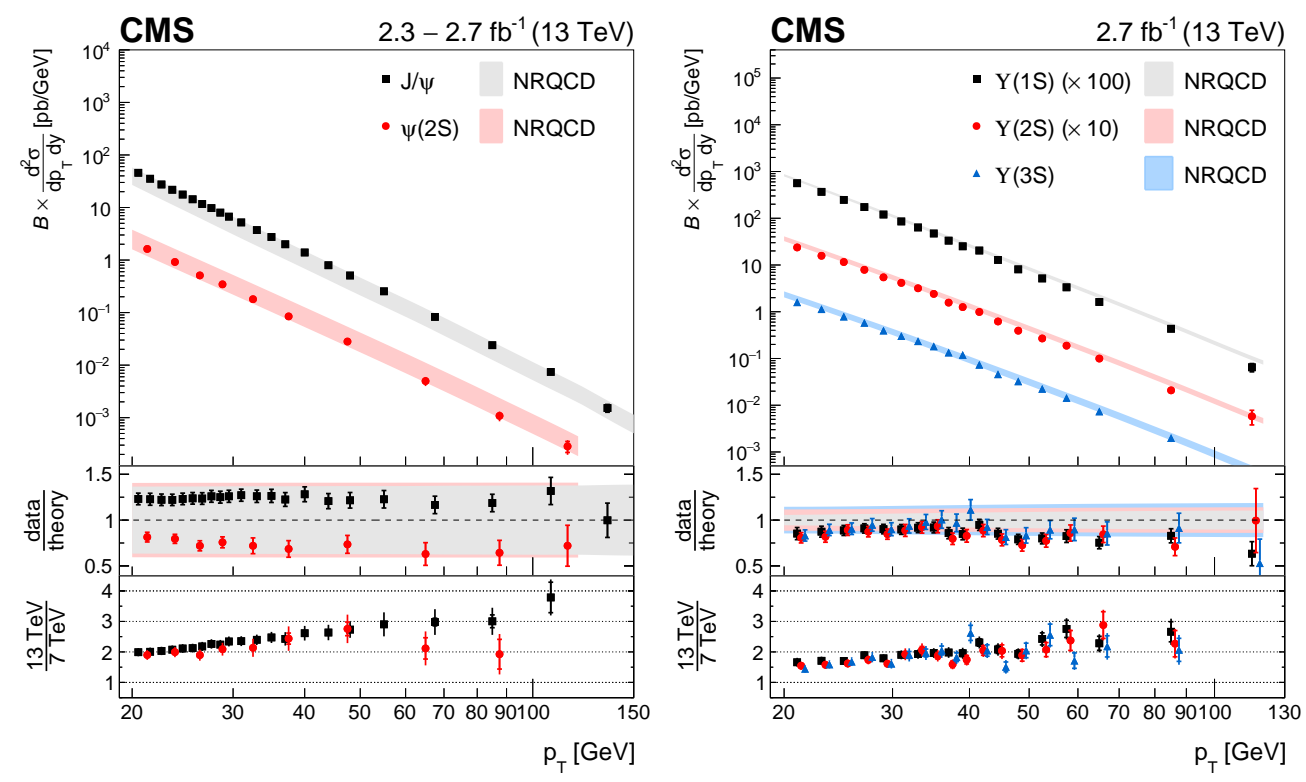

Figure 5: The production cross sections times branching fractions measured by CMS at $\sqrt{s}=13 \mathrm{TeV}$ for $\mathrm{J} / \psi$ and $\psi(2 \mathrm{~S})$ (left) and $\mathrm{Y}(n \mathrm{~S})$ (right) [26]. Ratios with respect to theoretical predictions [27, 28] and to CMS results at $\sqrt{s}=7 \mathrm{TeV}[29,30]$ are shown in the two bottom pads of each panel.

The analysis also extracts the ratios of cross sections as a function of $p_{\mathrm{T}}$ for $\psi(2 \mathrm{~S}), \mathrm{Y}(2 \mathrm{~S})$, and $\mathrm{Y}(1 \mathrm{~S})$ relative to their respective ground states. These are shown in Fig. 6. The prompt $\psi(2 \mathrm{~S})$ to $\mathrm{J} / \psi$ cross section ratio is constant as a function of $p_{\mathrm{T}}$, while the cross sections of the excited $\mathrm{Y}$ states relative to $\mathrm{Y}(1 \mathrm{~S})$ show a slight increase with $p_{\mathrm{T}}$. 


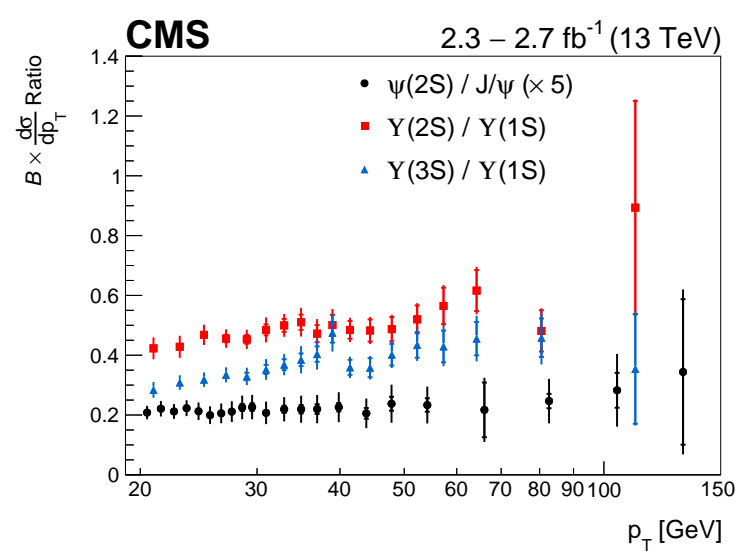

Figure 6: The $\psi(2 \mathrm{~S}) / \mathrm{J} / \psi$ (black), $\mathrm{Y}(2 \mathrm{~S}) / \mathrm{Y}(1 \mathrm{~S})$ (red), and $\mathrm{Y}(3 \mathrm{~S}) / \mathrm{Y}(1 \mathrm{~S})$ (blue) production cross section ratios as a function of $p_{\mathrm{T}}$ measured by CMS at $\sqrt{s}=13 \mathrm{TeV}$ [26].

\section{Conclusions}

Although the CMS detector is not currently taking new data due to the planned long shutdown 2 of the LHC, new measurements of heavy flavor production are still being published by the CMS Collaboration at a steady rate. These measurements are based on the more recent data at $\sqrt{s}=$ $13 \mathrm{TeV}$ from LHC Run-2 but also on the older data at $\sqrt{s}=7$ and $8 \mathrm{TeV}$ from LHC Run-1.

Among the most recent CMS results, these proceedings describe production cross section measurements for quarkonia and open beauty at $13 \mathrm{TeV}$, a study of $\mathrm{J} / \psi$ production inside jets, and a measurement of the $\Lambda_{\mathrm{b}}$ polarization at 7 and $8 \mathrm{TeV}$.

Other interesting results not treated here include a measurement of open beauty cross sections at $7 \mathrm{TeV}$ [31] and the first observations of the mass splits of the $\chi_{\mathrm{b} 1,2}(3 \mathrm{P})$ [32] and of the $\mathrm{B}_{\mathrm{c}}^{+}(2 \mathrm{~S})$ doublets [33]. The last measurement is described in the proceedings by L. Cristella.

\section{References}

[1] CMS Collaboration, The CMS experiment at the CERN LHC, JINST 3 (2008) S08004.

[2] CMS Collaboration, Measurement of the differential inclusive $B^{+}$hadron cross sections in pp collisions at $\sqrt{s}=13 \mathrm{TeV}$, Phys. Lett. B 771 (2017) 435 [1609.00873].

[3] CMS Collaboration, Measurement of the $B^{+}$Production Cross Section in pp Collisions at $\sqrt{s}=7 \mathrm{TeV}$, Phys. Rev. Lett. 106 (2011) 112001 [1101.0131].

[4] T. Sjöstrand, S. Mrenna, and P. Z. Skands, A Brief Introduction to PYTHIA 8.1, Comp. Phys. Comm. 178 (2008) 852 [0710.3820].

[5] M. Cacciari, M. Greco, and P. Nason, The $p_{T}$ spectrum in heavy-flavour hadroproduction, JHEP 05 (1998) 007 [hep-ph / 9803400$]$.

[6] M. Cacciari, S. Frixione, and P. Nason, The $p_{T}$ spectrum in heavy-flavour photoproduction, JHEP 03 (2001) 006 [hep-ph/ 0102134$]$.

[7] CMS Collaboration, Measurement of the $\Lambda_{b}$ polarization and angular parameters in $\Lambda_{b} \rightarrow J / \psi \Lambda$ decays from pp collisions at $\sqrt{s}=7$ and 8TeV, Phys. Rev. D 97 (2018) 072010 [1802. 04867]. 
[8] O. Leitner, Z. J. Ajaltouni, and E. Conte, An angular distribution analysis of $\Lambda_{b}$ decays, Nucl. Phys. A 755 (2005) 435 [hep-ph/ 0412131$].$

[9] Z. J. Ajaltouni, E. Conte, and O. Leitner $\Lambda_{b}$ decays into $\Lambda$-vector, Phys. Lett. B 614 (2005) 165 [hep-ph/0412116].

[10] T. Gutsche, M. A. Ivanov, J. G. Körner, V. E. Lyubovitskij, and P. Santorelli, Polarization effects in the cascade decay $\Lambda_{b} \rightarrow \Lambda\left(\rightarrow p \pi^{-}\right)+J / \psi\left(\rightarrow \ell^{+} \ell^{-}\right)$in the covariant confined quark model, Phys. Rev. D 88 (2013) 114018 [1309. 7879].

[11] H.-Y. Cheng, Nonleptonic weak decays of bottom baryons, Phys. Rev. D 56 (1997) 2799 (erratum Phys. Rev. D 99 (2019) 079901) [hep-ph / 9612223 ].

[12] Fayyazuddin and Riazuddin, Two body nonleptonic $\Lambda_{b}$ decays in the quark model with a factorization ansatz, Phys. Rev. D 58 (1998) 014016 [hep-ph / 9802326$].$

[13] R. Mohanta, A. K. Giri, M. P. Khanna, M. Ishida, S. Ishida, and M. Oda, Hadronic Weak Decays of $\Lambda_{b}$ Baryon in the Covariant Oscillator Quark Model, Prog. Theor. Phys. 101 (1999) 959 [hep-ph/9904324].

[14] Z.-T. Wei, H.-W. Ke, and X.-Q. Li, Evaluating decay rates and asymmetries of $\Lambda_{b}$ into light baryons in the light-front quark model, Phys. Rev. D 80 (2009) 094016 [0909.0100].

[15] C.-H. Chou, H.-H. Shih, S.-C. Lee, and H.-n. Li, $\Lambda_{b} \rightarrow \Lambda J / \psi$ decay in perturbative QCD, Phys. Rev. D 65 (2002) 074030 [hep-ph/ 0112145$].$

[16] LHCb Collaboration, Measurements of the $\Lambda_{b}^{0} \rightarrow J / \psi \Lambda$ decay amplitudes and the $\Lambda_{b}^{0}$ polarisation in pp collisions at $\sqrt{s}=7$ TeV, Phys. Lett. B 724 (2013) 27 [1302.5578].

[17] ATLAS Collaboration, Measurement of the parity-violating asymmetry parameter $\alpha_{b}$ and the helicity amplitudes for the decay $\Lambda_{b}^{0} \rightarrow J / \psi \Lambda^{0}$ with the ATLAS detector, Phys. Rev. D 89 (2014) 092009 [1404.1071].

[18] CMS Collaboration, Measurements of correlations between $J / \psi$ mesons and jets produced in $\sqrt{s}=8 T e V$ pp collisions, CMS-PAS-BPH-15-003, [http://cds.cern.ch/record/2674046].

[19] CMS Collaboration, Study of $J / \psi$ meson production from jet fragmentation in pp collisions at $\sqrt{s}=8 \mathrm{TeV}$, submitted to Phys. Lett. B, 1910.01686.

[20] T. Adye, Unfolding algorithms and tests using RooUnfold, in Proceedings, PHYSTAT 2011 Workshop on Statistical Issues Related to Discovery Claims in Search Experiments and Unfolding (2011) 313 [1105.1160].

[21] M. Baumgart, A. K. Leibovich, T. Mehen, and I. Z. Rothstein, Probing quarkonium production mechanisms with jet substructure, JHEP 1411 (2014) 003 [1406.2295].

[22] G. T. Bodwin, H. S. Chung, U.-R. Kim, and J. Lee, Fragmentation contributions to J/ $\psi$ production at the Tevatron and the LHC, Phys. Rev. Lett. 113 (2014) 022001 [1403. 3612].

[23] M. Butenschoen and B. A. Kniehl, Next-to-leading-order tests of NRQCD factorization with J/ $\psi$ yield and polarization, Mod. Phys. Lett. A 28 (2013) 1350027 [1212 . 2037].

[24] CDF Collaboration, Polarizations of $J / \psi$ and $\psi(2 S)$ mesons produced in $p \bar{p}$ collisions at $\sqrt{s}=1.96$ TeV, Phys. Rev. Lett. 99 (2007) 132001

[25] CMS Collaboration, Measurement of the prompt $J / \psi$ and $\psi(2 S)$ polarizations in pp collisions at $\sqrt{s}=7 \mathrm{TeV}$, Phys. Lett. B 727 (2013) 381 [1307 . 6070]. 
[26] CMS Collaboration, Measurement of quarkonium production cross sections in pp collisions at $\sqrt{s}=13 \mathrm{TeV}$, Phys. Lett. B $\mathbf{7 8 0}$ (2018) 251 [1710.11002].

[27] Y.-Q. Ma, K. Wang, and K.-T. Chao, $J / \psi\left(\psi^{\prime}\right)$ Production at the Tevatron and LHC at $\mathscr{O}\left(\alpha_{s}^{4} v^{4}\right)$ in nonrelativistic QCD, Phys. Rev. Lett. 106 (2011) 042002 [1009. 3655].

[28] H. Han, Y.-Q. Ma, C. Meng, H.-S. Shao, Y.-J. Zhang, and K.-T. Chao, $Y(n S)$ and $\chi_{b}(n P)$ production at hadron colliders in nonrelativistic QCD, Phys. Rev. D 94 (2016) 014028 [1410.8537].

[29] CMS Collaboration, Measurement of $J / \psi$ and $\psi(2 S)$ prompt double-differential cross sections in pp collisions at $\sqrt{s}=7$ TeV, Phys. Rev. Lett. 114 (2015) 191802 [1502. 04155$].$

[30] CMS Collaboration, Measurements of the $Y(1 S), Y(2 S)$, and $Y(3 S)$ differential cross sections in pp collisions at $\sqrt{s}=7 \mathrm{TeV}$, Phys. Lett. B 749 (2015) 14 [1501.07750].

[31] CMS Collaboration, Measurement of production cross sections times branching fraction of $B_{c}^{+} \rightarrow J / \psi \pi^{+}$and $B^{+} \rightarrow J / \psi K^{+}$in pp collisions at $\sqrt{s}=7$ TeV at CMS, CMS-PAS-BPH-13-002, [http://cds.cern.ch/record/2621286].

[32] CMS Collaboration, Observation of the $\chi_{b 1}(3 P)$ and $\chi_{b 2}(3 P)$ and measurement of their masses, Phys. Rev. Lett. 121 (2018) 092002 [1805.11192].

[33] CMS Collaboration, Observation of two excited $B_{c}^{+}$states and measurement of the $B_{c}^{+}(2 S)$ mass in pp collisions at $\sqrt{s}=13$ TeV, Phys. Rev. Lett. 122 (2019) 132001 [1902.00571]. 